13

\title{
Неустойчивость трубчатого электронного пучка, движущегося над диэлектрическим цилиндром
}

\author{
(С Ю.О. Аверков, Ю.В. Прокопенко, В.М. Яковенко
}

Институт радиофизики и электроники им. А.Я. Усикова НАН Украины, 61085 Харьков, Украина

e-mail: yuriyaverkov@gmail.com

(Поступило в Редакцию 28 июля 2016 г. В окончательной редакции 4 апреля 2017 г.)

Теоретически изучен механизм взаимодействия собственных волн диэлектрического цилиндра с нерелятивистским трубчатым электронным пучком, движущимся над его поверхностью. Получено дисперсионное уравнение связанных волн электронов пучка и диэлектрического цилиндра. Сформулированы условия их неустойчивости и найдены инкременты. Анализ дисперсионного уравнения и выражений для инкрементов показывает, что наиболее эффективно возбуждаются волны электрического типа с большими азимутальными индексами при малых величинах зазора между пучком и цилиндром.

DOI: 10.21883/JTF.2017.10.45003.2015

\section{Введение}

При взаимодействии электронного пучка с различными электродинамическими структурами возникают неустойчивости электромагнитных волн. В основе механизмов неустойчивостей лежат эффекты Вавилова-Черенкова, циклотронного излучения и Допплера [1-4]. Наиболее полное и последовательное изложение эффектов взаимодействия потоков заряженных частиц с плазменными средами различных конфигураций представлено в [3]. В частности, в [3,4] приведены результаты исследований плазменных релятивистских генераторов и усилителей, в которых трубчатый электронный поток транспортируется над трубчатым плазменным цилиндром, расположенным в соосном внешнем магнитном поле. В работе [5] было экспериментально обнаружено излучение электромагнитных волн системой, в которой многоструйный поток электронов пролетает над диэлектрическим цилиндром. Для понимания механизма генерирования, безусловно, заслуживает внимания теоретическое исследование взаимодействия трубчатого потока электронов с диэлектрическим цилиндром. Этой теме и посвящается настоящая работа. Предполагается, что в азимутальном направлении поток частиц является сплошным. Мы показываем, что в такой системе возникает неустойчивость объемно-поверхностных электромагнитных волн, обусловленная черенковским механизмом взаимодействия электромагнитного поля и заряженных частиц. Найдены инкременты неустойчивости.

\section{Постановка задачи, основные уравнения и граничные условия}

Пусть диэлектрический цилиндр с радиусом $\rho_{0}$ занимает область пространства $0 \leq \rho \leq \rho_{0}, 0 \leq \varphi \leq 2 \pi$ и $-\infty \leq z \leq+\infty$ (рис. 1). Цилиндр изготовлен из изотропного немагнитного материала, диэлектрическая проницаемость которого равна $\varepsilon$. На расстоянии $\rho_{b}$ от оси цилиндра в вакууме транспортируется трубчатый поток электронов с радиальной толщиной $a$ и плотностью $N_{0}(\rho)$. Электроны движутся со скоростью $v_{0}$. Предполагается, что заряд электронов компенсируется положительным фоном заряженных частиц, а толщина потока $a$ мала по сравнению с другими пространственными масштабами электродинамической системы. Следовательно, равновесную плотность электронов в трубчатом пучке можно представить в виде $N_{0}(\rho)=N_{0} a \delta\left(\rho-\rho_{b}\right)$, где $N_{0}-$ равновесная концентрация электронов пучка, $\delta\left(\rho-\rho_{b}\right)$ - дельта-функция Дирака. В линеаризованном виде плотность тока пучка в точке с радиусом-вектором $\mathbf{r}$ в момент времени $t$ имеет вид

$$
\mathbf{j}(\mathbf{r}, t)=e N_{0}(\rho) \mathbf{v}(\mathbf{r}, t)+e \mathbf{v}_{0} N(\mathbf{r}, t)
$$

где $e$ - заряд электрона, $N(\mathbf{r}, t)$ и $\mathbf{v}(\mathbf{r}, t)$ - переменные составляющие плотности и скорости электронов пучка, $\mathbf{v}_{0}=v_{0} \mathbf{e}_{z}\left(\mathbf{e}_{z}-\right.$ орт оси $\left.Z\right)$. В дальнейшем радиальную компоненту плотности тока пучка полагаем равной нулю в силу выбранной модели бесконечно тонкого пучка.

Система уравнений, описывающая взаимодействие электронного пучка с собственными колебаниями диэлектрического цилиндра, представляет собой уравнения Максвелла, дополненные линеаризованными уравне-

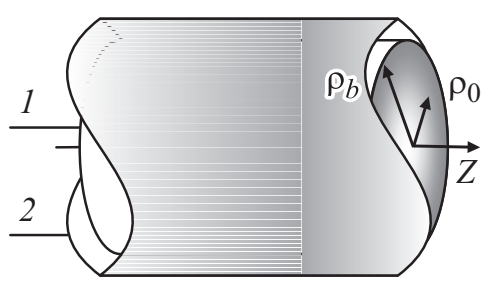

Рис. 1. Электродинамическая система. 1 - диэлектрический цилиндр, 2 - трубчатый электронный пучок. 
ниями непрерывности и движения для электронов пучка:

$$
\begin{gathered}
\operatorname{rot} \mathbf{H}(\mathbf{r}, t)-\frac{1}{c} \frac{\partial}{\partial t} \mathbf{D}(\mathbf{r}, t)=\frac{4 \pi}{c} \mathbf{j}(\mathbf{r}, t) \\
\operatorname{rot} \mathbf{E}(\mathbf{r}, t)+\frac{1}{c} \frac{\partial}{\partial t} \mathbf{H}(\mathbf{r}, t)=0 \\
\operatorname{div} \mathbf{D}(\mathbf{r}, t)=4 \pi e N(\mathbf{r}, t) \\
\operatorname{div} \mathbf{H}(\mathbf{r}, t)=0 \\
e \frac{\partial N(\mathbf{r}, t)}{\partial t}+\operatorname{div} \mathbf{j}(\mathbf{r}, t)=0 \\
\frac{\partial \mathbf{v}(\mathbf{r}, t)}{\partial t}+v_{0} \frac{\partial \mathbf{v}(\mathbf{r}, t)}{\partial z}=\frac{e}{m}\left(\mathbf{E}(\mathbf{r}, t)+\frac{1}{c}\left[\mathbf{v}_{0}, \mathbf{H}(\mathbf{r}, t)\right]\right)
\end{gathered}
$$

где $m$ - масса электрона, $\mathbf{H}(\mathbf{r}, t)$ - напряженность магнитного поля, вектор индукции электрического поля $\mathbf{D}(\mathbf{r}, t)$ связан с вектором напряженности $\mathbf{E}(\mathbf{r}, t)$ материальным уравнением $\mathbf{D}(\mathbf{r}, t)=\varepsilon \mathbf{E}(\mathbf{r}, t)$.

Для нахождения дисперсионного уравнения колебаний в рассматриваемой системе необходимо воспользоваться следующими граничными условиями для электрических и магнитных полей при $\rho=\rho_{0}$ и $\rho=\rho_{b}$ :

- при $\rho=\rho_{0}$ непрерывны тангенциальные составляющие электрического и магнитного полей,

- при $\rho=\rho_{b}$ непрерывны тангенциальные составляющие электрического поля, а тангенциальные составляющие магнитного поля и нормальная компонента электрической индукции поля терпят разрывы, вызванные возмущенным током и зарядом пучка соответственно.

Условия разрывов тангенциальных компонент магнитного поля $\left(H_{\varphi}(\mathbf{r}, t)\right.$ и $\left.H_{z}(\mathbf{r}, t)\right)$ и нормальной компоненты электрической индукции (в вакууме $D_{\rho}(\mathbf{r}, t) \equiv$ $\left.\equiv E_{\rho}(\mathbf{r}, t)\right)$ получаются путем интегрирования (1) и (3) по бесконечно малой толщине пучка. Эти условия имеют вид

$$
\begin{aligned}
\left.H_{\varphi}(\mathbf{r}, t)\right|_{\rho=\rho_{b}+0}- & \left.H_{\varphi}(\mathbf{r}, t)\right|_{\rho=\rho_{b}-0} \\
& =\frac{4 \pi}{c \rho_{b}} \lim _{\Delta \rho \rightarrow 0} \int_{\rho_{b}-\Delta \rho}^{\rho_{b}+\Delta \rho} j_{z}(\mathbf{r}, t) \rho d \rho, \\
\left.H_{z}(\mathbf{r}, t)\right|_{\rho=\rho_{b}+0}- & \left.H_{z}(\mathbf{r}, t)\right|_{\rho=\rho_{b}-0} \\
& =-\frac{4 \pi}{c} \lim _{\Delta \rho \rightarrow 0} \int_{\rho_{b}-\Delta \rho}^{\rho_{b}+\Delta \rho} j_{\varphi}(\mathbf{r}, t) d \rho, \\
\left.E_{\rho}(\mathbf{r}, t)\right|_{\rho=\rho_{b}+0}- & \left.E_{\rho}(\mathbf{r}, t)\right|_{\rho=\rho_{b}-0} \\
& =\frac{4 \pi e}{\rho_{b}} \lim _{\Delta \rho \rightarrow 0} \int_{\rho_{b}-\Delta \rho} N(\mathbf{r}, t) \rho d \rho,
\end{aligned}
$$

где $j_{\varphi}(\mathbf{r}, t)$ и $j_{z}(\mathbf{r}, t)$ - азимутальная и аксиальная составляющие плотности тока пучка $\mathbf{j}(\mathbf{r}, t)$.
Представим все переменные величины в виде набора пространственно-временны́х гармоник, например:

$$
\mathbf{E}(\mathbf{r}, t)=\sum_{m=-\infty}^{\infty} \int_{-\infty}^{\infty} \int_{-\infty}^{\infty} \mathbf{E}_{n}\left(\rho, q_{z}, \omega\right) e^{i\left(q_{z} z+n \varphi-\omega t\right)} d q_{z} d \omega,
$$

где $\omega, q_{z}$ и $n-$ частота, продольное волновое число и номер пространственной гармоники (совпадающий с азимутальным модовым индексом) волны соответственно.

С учетом представлений переменных величин в виде пространственно-временнб́х гармоник (подобно (5)) исходные уравнения (1)-(4) вне электронного пучка $\left(\rho \neq \rho_{b}\right)$ для аксиальных спектральных составляющих поля приобретают вид

$$
\left[\frac{1}{\rho} \frac{\partial}{\partial \rho} \rho \frac{\partial}{\partial \rho}+\left(q_{v}^{2}-\frac{n^{2}}{\rho^{2}}\right)\right]\left\{\begin{array}{l}
E_{z n}\left(\rho, q_{z}, \omega\right) \\
H_{z n}\left(\rho, q_{z}, \omega\right)
\end{array}=0\right\},
$$

где $v=1,2 ; q_{v}^{2}=\varepsilon_{v} \omega^{2} / c^{2}-q_{z}^{2}-$ квадрат поперечного волнового числа электромагнитных волн, $\varepsilon_{1}=\varepsilon$ (цилиндр) и $\varepsilon_{2}=1$ (вакуум). Когда $q_{v}^{2}>0$, выражения (6) имеют вид уравнений Бесселя, а при $q_{v}^{2}<0-$ модифицированных уравнений Бесселя. Рассмотрим случай $q_{2}^{2}<0$, поскольку, как будет позже показано, для волн, создаваемых заряженными частицами, имеем $q_{z}=\omega / v_{0}$ и $\omega^{2} / c^{2} \ll q_{z}^{2}$, так как $v_{0} \ll c$. Следовательно, вне трубчатого электронного пучка спектральные составляющие электромагнитного поля $E_{z n}\left(\rho, q_{z}, \omega\right)$ и $H_{z n}\left(\rho, q_{z}, \omega\right)$ имеют вид

$$
\begin{aligned}
& E_{z n}\left(\rho, q_{z}, \omega\right)=
\end{aligned}
$$

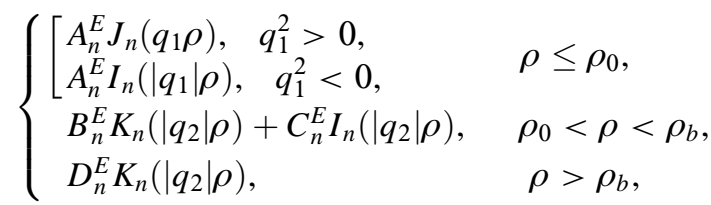

$$
\begin{aligned}
& H_{z n}\left(\rho, q_{z}, \omega\right)=
\end{aligned}
$$

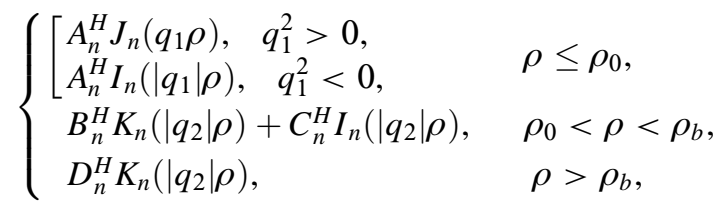

где $J_{n}(u)$ - функция Бесселя $n$-го порядка первого рода и одноименные модифицированные функции первого рода - $I_{n}(u)$ (функция Инфельда) и второго рода $K_{n}(u)$ (функция Макдональда) $[6] ; A_{n}^{E, H}, B_{n}^{E, H}, C_{n}^{E, H}$ и $D_{n}^{E, H}$ - произвольные постоянные. Выбор решения обусловлен выполнением условий конечности величин $E_{z n}\left(\rho, q_{z}, \omega\right)$ и $H_{z n}\left(\rho, q_{z}, \omega\right)$ при $\rho \rightarrow 0 \quad$ и $\rho \rightarrow \infty$. Можно показать, что при $v_{0}<c / \sqrt{\varepsilon}$ выражения для $E_{z n}\left(\rho, q_{z}, \omega\right)$ - и $H_{z n}\left(\rho, q_{z}, \omega\right)$-компонент поля внутри цилиндра описываются модифицированными функциями Бесселя 1-го рода, а при $v_{0}>c / \sqrt{\varepsilon}-$ функциями 
Бесселя. В дальнейшем мы рассмотрим случай, когда $v_{0}>c / \sqrt{\varepsilon}$ (условие эффекта Вавилова-Черенкова). Остальные фурье-компоненты электромагнитных полей в области цилиндра $\left(\rho<\rho_{0}\right)$, зазора $\left(\rho_{0}<\rho<\rho_{b}\right)$ и за пучком $\left(\rho>\rho_{0}\right)$ выражаем с помощью уравнений Максвелла через величины $E_{z n}\left(\rho, q_{z}, \omega\right)$ и $H_{z n}\left(\rho, q_{z}, \omega\right)$.

Полагая пучок нерелятивистским и удовлетворяя указанным ранее граничным условиям на поверхности цилиндра и электронного пучка, получим следующее дисперсионное уравнение для связанных колебаний диэлектрического цилиндра и пучка:

$$
\begin{array}{r}
\Delta\left(\omega-q_{z} v_{0}\right)^{2}=\Omega^{2}\left(n^{2}+q_{z}^{2} \rho_{b}^{2}\right) K_{n}^{2}\left(\left|q_{z}\right| \rho_{b}\right) \\
\times\left(\Delta \frac{I_{n}\left(\left|q_{z}\right| \rho_{b}\right)}{K_{n}\left(\left|q_{z}\right| \rho_{b}\right)}-\Delta_{1} \frac{I_{n}\left(\left|q_{z}\right| \rho_{0}\right)}{K_{n}\left(\left|q_{z}\right| \rho_{0}\right)}\right),
\end{array}
$$

где $\Omega^{2}=\omega_{b}^{2} a / \rho_{b}, \omega_{b}=\sqrt{4 \pi e^{2} N_{0} / m}-$ плазменная частота электронов пучка;

$$
\begin{gathered}
\Delta=\Delta_{0}-\Delta^{E} \Delta^{H} ; \quad \Delta_{0}=\left[\frac{n \omega(\varepsilon-1)}{q_{z} q_{1}^{2} \rho_{0}^{2} c}\right]^{2} ; \\
\Delta^{E}=\frac{1}{\left|q_{z}\right| \rho_{0}} \frac{K_{n}^{\prime}\left(\left|q_{z}\right| \rho_{0}\right)}{K_{n}\left(\left|q_{z}\right| \rho_{0}\right)}+\frac{\varepsilon}{q_{1} \rho_{0}} \frac{J_{n}^{\prime}\left(q_{1} \rho_{0}\right)}{J_{n}\left(q_{1} \rho_{0}\right)} ; \\
\Delta^{H}=\frac{1}{\left|q_{z}\right| \rho_{0}} \frac{K_{n}^{\prime}\left(\left|q_{z}\right| \rho_{0}\right)}{K_{n}\left(\left|q_{z}\right| \rho_{0}\right)}+\frac{1}{q_{1} \rho_{0}} \frac{J_{n}^{\prime}\left(q_{1} \rho_{0}\right)}{J_{n}\left(q_{1} \rho_{0}\right)} ; \\
\Delta_{1}=\Delta_{0}-\left(\frac{1}{\left|q_{z}\right| \rho_{0}} \frac{I_{n}^{\prime}\left(\left|q_{z}\right| \rho_{0}\right)}{I_{n}\left(\left|q_{z}\right| \rho_{0}\right)}+\frac{\varepsilon}{q_{1} \rho_{0}} \frac{J_{n}^{\prime}\left(q_{1} \rho_{0}\right)}{J_{n}\left(q_{1} \rho_{0}\right)}\right) \Delta^{H} .
\end{gathered}
$$

В отсутствие электронного пучка $(\Omega=0)$ выражение (7) представляет собой дисперсионное уравнение объемно-поверхностных волн в диэлектрическом цилиндре. В случае изотропного плазменного цилиндра (при отсутствии внешнего магнитного поля), когда $q_{1}^{2}<0$, выражение (7) представляет собой дисперсионное уравнение поверхностных волн. Для симметричных волн $(n=0) E$-типа оно принимает вид, приведенный в книгах $[3,4]$.

$\mathrm{B}$ настоящей работе рассматривается взаимодействие движущихся заряженных частиц и объемно-поверхностных волн диэлектрического цилиндра.

При $\rho_{0}=\rho_{b}$ и $v_{0}=0$ формула (7) имеет вид дисперсионного уравнения диэлектрического цилиндра с $2 D$-плазменным слоем на его боковой поверхности и при $c \rightarrow \infty$ это уравнение приведено в [7-10]. В случае отсутствия диэлектрического волновода $(\varepsilon=1)$ и $v_{0}=0$ уравнение (7) при $c \rightarrow \infty$ принимает вид дисперсионного уравнения „нанотрубки“ $[11]$.

Для пучка электронов с малой плотностью решениями уравнения (7) являются точки пересечения прямой $\omega=q_{z} v_{0}$ и дисперсионных кривых диэлектрического цилиндра $\Delta=0$, имеющие координаты $\left(q_{z n s}, \omega_{n s}\right)$, где $n$ и $s$ - азимутальный и радиальный индексы. При этом квадраты поперечных волновых чисел имеют значения $q_{1}^{2}=\omega_{n s}\left(\varepsilon \beta^{2}-1\right) / v_{0}^{2}$, где $\beta=v_{0} / c$. В цилиндре существуют собственные гибридные $\mathrm{HE}$ ( $E$-типа)- и
ЕН (H-типа)-колебания [12]. При прохождении пучка моноэнергетических электронов вблизи поверхности диэлектрического цилиндра электроны потока, находящиеся в фазе с собственной волной цилиндра, для которых $q_{z} v_{0} \approx \omega_{n s}$, могут раскачивать колебания НЕ- и ЕН-ветвей. При этом каждая резонансная частица пучка возбуждает собственные колебания цилиндра независимо. Можно показать, что инкременты неустойчивости для НЕ-волн больше, чем для ЕН-волн.

Положим $\omega=\omega_{n s}+\delta \omega$, где $\omega_{n s}=q_{z n s} v_{0}$ и $\mid \delta \omega /$ $\omega_{n s} \mid \ll 1[13]$. Подставив $\omega=\omega_{n s}+\delta \omega$ в дисперсионное уравнение (7) и пренебрегая малыми порядка $O\left\lfloor(\delta \omega)^{4}\right\rfloor$, получим следующее кубическое уравнение относительно $\delta \omega$ :

$$
\begin{aligned}
(\delta \omega)^{3}= & \Omega^{2}\left(n^{2}+q_{z n s}^{2} \rho_{b}^{2}\right) K_{n}^{2}\left(\left|q_{z n s}\right| \rho_{b}\right) \\
& \times\left.\frac{I_{n}\left(\left|q_{z n s}\right| \rho_{0}\right)}{K_{n}\left(\left|q_{z n s}\right| \rho_{0}\right)} \Delta_{1}\right|_{\omega=\omega_{n s}}\left(\left.\frac{\partial \Delta}{\partial \omega}\right|_{\omega=\omega_{n s}}\right)^{-1} .
\end{aligned}
$$

Отсюда следует, что при резонансе частота одной из связанных волн увеличивается относительно $\omega_{n s}$ на величину $\operatorname{Re} \delta \omega=|\delta \omega|^{1 / 3}$, а ее фазовая скорость $\left(\omega_{n s}+|\delta \omega|^{1 / 3}\right) / q_{z n s}$ превышает скорость электронов пучка. В системе осуществляется устойчивое взаимодействие волн электронов пучка и собственной волны цилиндра. В результате возникает быстрая (высокочастотная) связанная волна. Частоты двух других связанных волн уменьшаются на величину $\operatorname{Re} \delta \omega=|\delta \omega|^{1 / 3} / 2$, а их фазовые скорости $\left(\omega_{n s}-|\delta \omega|^{1 / 3} / 2\right) / q_{z n s}<v_{0}$. Эти волны являются медленными (низкочастотными) относительно скорости электронов. Одна из этих двух волн характеризуется положительной мнимой добавкой к частоте $\operatorname{Im} \delta \omega=\sqrt{3}|\delta \omega|^{1 / 3} / 2$ и, следовательно, является неустойчивой во времени - амплитуда этой волны, согласно (5), растет экспоненциально. При этом амплитуда другой волны экспоненциально убывает. На диаграмме $\omega\left(q_{z}\right)$ области неустойчивости отображаются в виде разрывов дисперсионных ветвей взаимодействующих волн. Поскольку инкремент возбуждаемых волн пропорционален $N_{0}^{1 / 3}$, возбуждение волн резонансными частицами пучка осуществляется когерентно [13].

\section{Численный анализ дисперсионного уравнения и распределений полей связанных волн}

Заслуживает внимания численный анализ дисперсионного уравнения (7). В качестве материала диэлектрического цилиндра выберем поликор с $\varepsilon=9.6$, а радиус цилиндра $\rho_{0}=1 \mathrm{~cm}$. Радиус трубчатого электронного пучка $\rho_{b}=1.2 \mathrm{~cm}$. Значения равновесной концентрации электронов пучка $N_{0}$, толщины стенки пучка $a$ и скорости направленного движения электронов пучка $v_{0}$ выберем следующими: $N_{0}=5.9 \cdot 10^{10} \mathrm{~cm}^{-3}, a=0.05 \mathrm{~cm}$ и $v_{0}=0.387 c$ соответственно. 

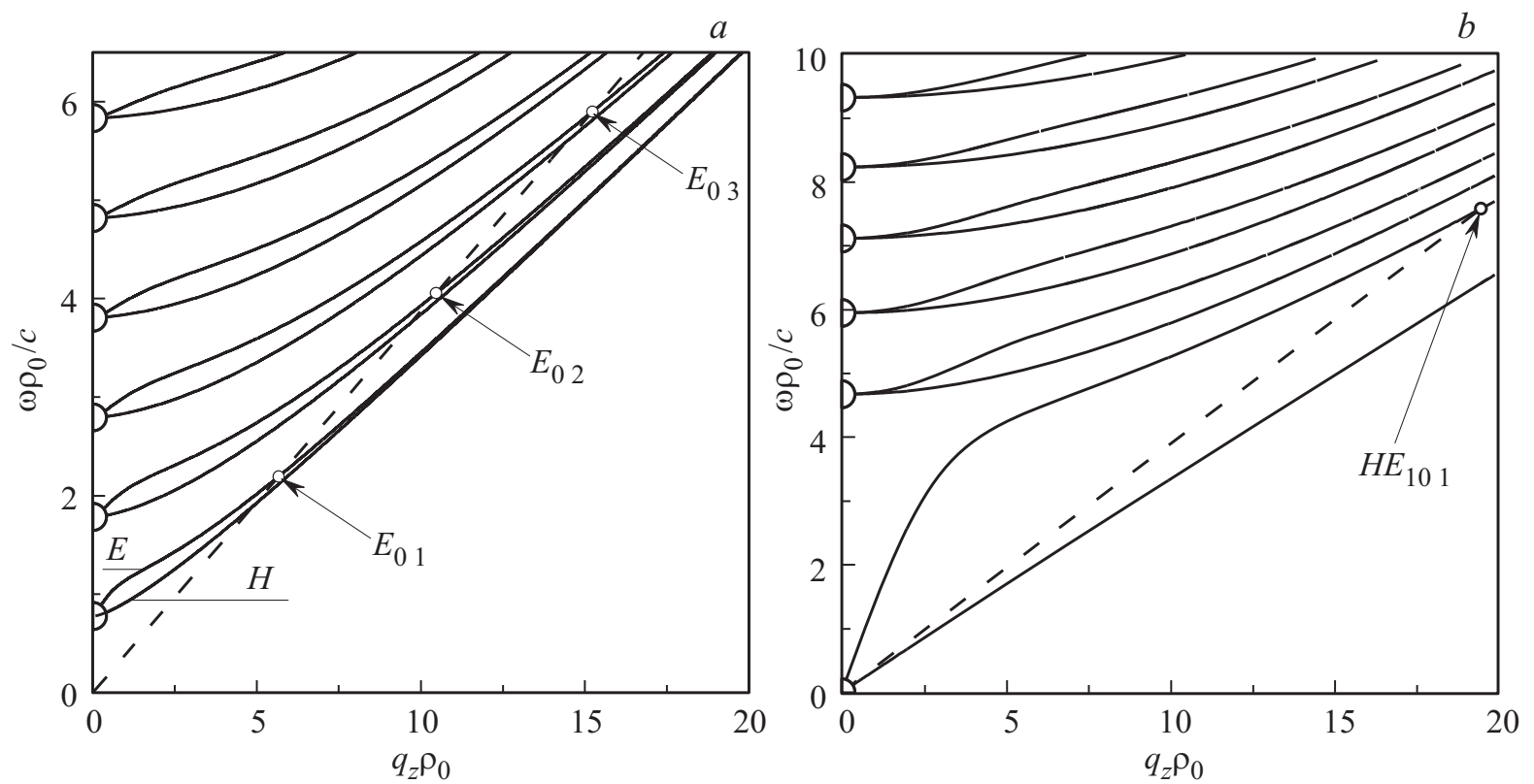

Рис. 2. Черенковское взаимодействие электронного пучка с собственными симметричными $(a)$ и несимметричными $(b)$ модами диэлектрического цилиндра.
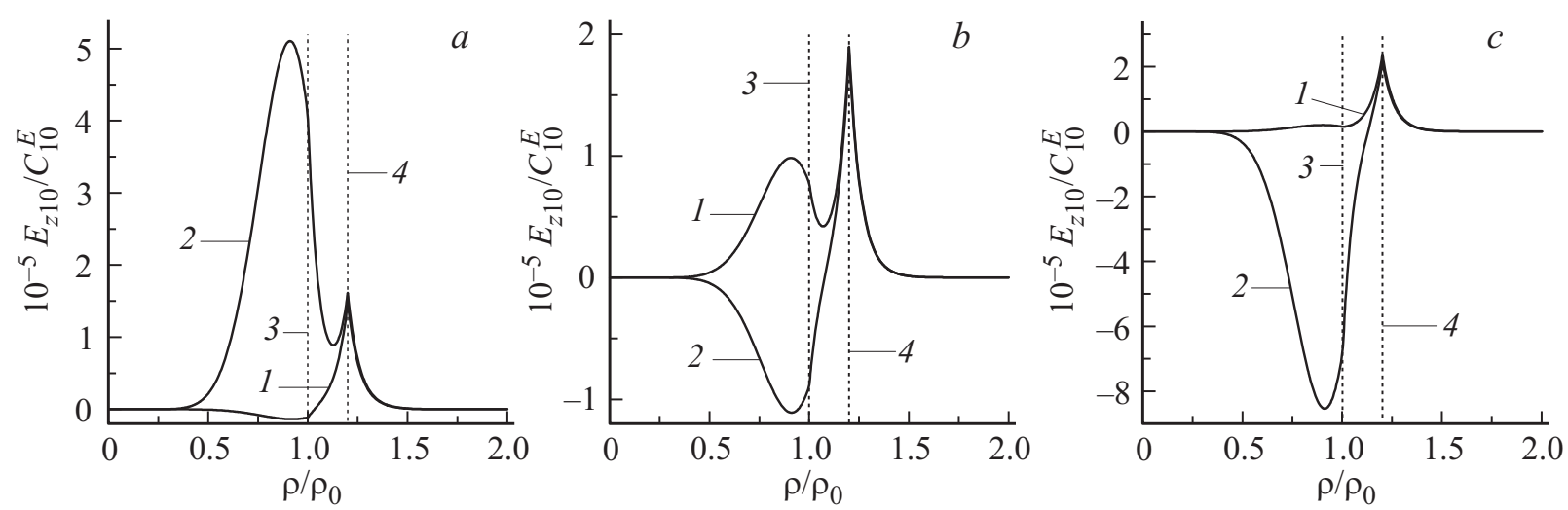

Рис. 3. Радиальное распределение $E_{z 101}$-компонент полей волны электрона пучка $(1)$ и собственной $\mathrm{HE}_{101}$-моды цилиндра $(2)$ в областях: $a-q_{z}<q_{z 101} ; b-q_{z}=q_{z 101} ; c-q_{z}>q_{z 101} .3-\rho=\rho_{0}$ (поверхность цилиндра), $4-\rho=\rho_{b}$ (поверхность пучка).

На рис. 2 приведены дисперсионные зависимости собственных симметричных $(n=0)$ и несимметричных $(n \neq 0)$ мод диэлектрического цилиндра, представляющие решения уравнения $\Delta=0$. Штриховая прямая соответствует частотам волны электрона $\omega=q_{z} v_{0}$.

Из рис. 2, $a$ видно, что нижние границы частотных спектров симметричных мод цилиндра, отличающихся радиальным индексом $s$, определяются частотами отсечек при $q_{z}=0$. Из каждой точки начал спектров выходит пара дисперсионных кривых. Нижние кривые в таких парах соответствуют $H$-волнам, спектр которых получается из решения дисперсионного уравнения $\Delta^{H}=0$, а верхние кривые $-E$-волнам, которые являются решениями уравнения $\Delta^{E}=0$. Отметим, что при $n=0$ дисперсионное уравнение $\Delta=0$ распадается на два независимых уравнения $\Delta^{E}=0$ и $\Delta^{H}=0$. Радиальный модовый индекс $s$ определяется порядковым номером корня соответствующего дисперсионного уравнения и соответствует количеству вариаций поля вдоль радиальной координаты [12]. Составляющие электромагнитного поля $E$-волн имеют три компоненты $-E_{\rho n s}, H_{\varphi n s}$ и $E_{z n s}$, а $H$-волн $-H_{\rho n s}, E_{\varphi n s}$ и $H_{z n s}$.

На рис. $2, b$ представлены дисперсионные зависимости собственных мод цилиндра с азимутальным индексом $n=10$. Такие моды относятся к модам „шепчущей галереи“", представляющим большой практический интеpec [12]. Отметим, что электромагнитные поля несимметричных мод цилиндра содержат все шесть компонент, за исключением аксиально-однородных мод, у которых $q_{z}=0$. Нижние ветви каждой пары дисперсионных зависимостей цилиндра, которые объединены частотой отсечки, соответствуют частотам собственных 

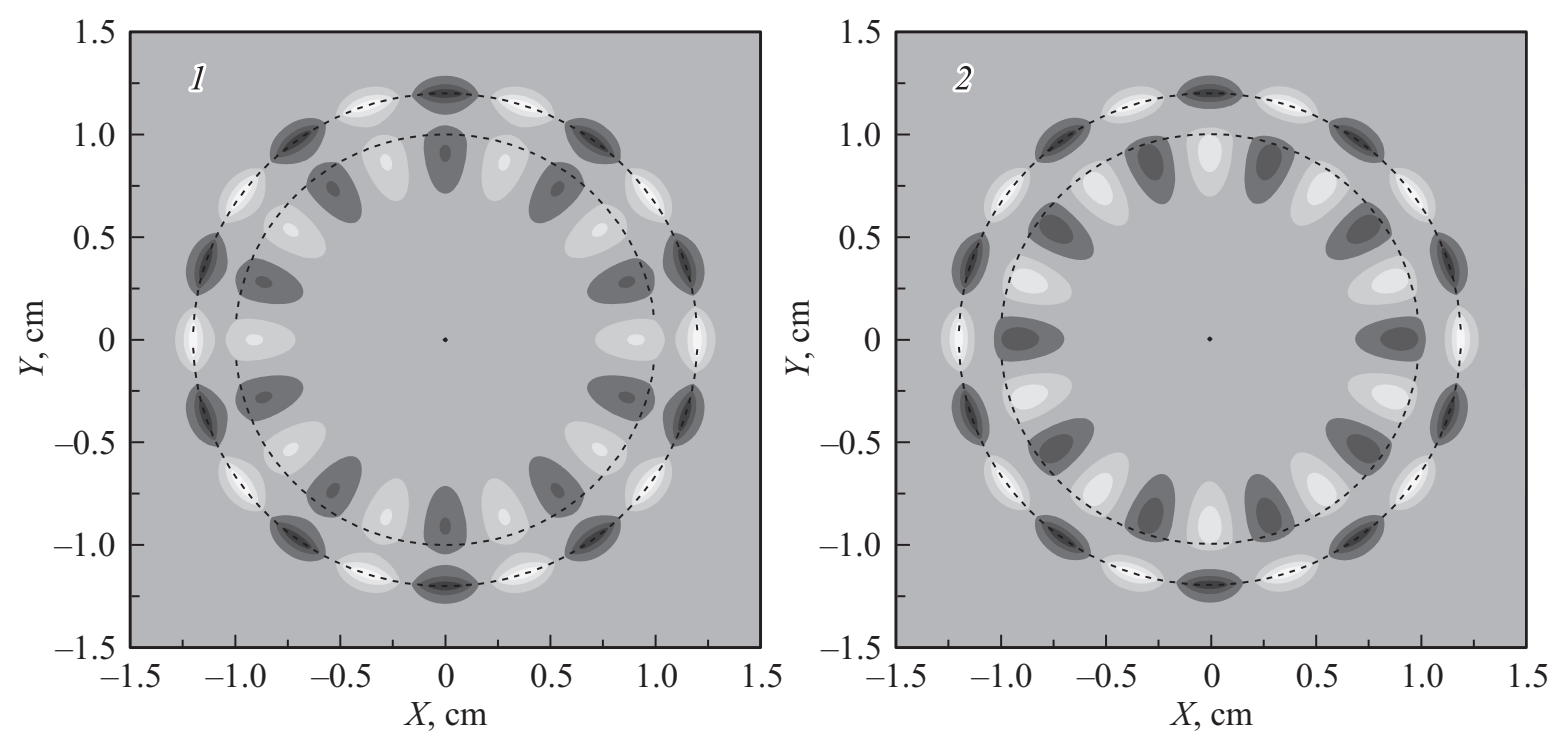

Рис. 4. Распределение аксиальной компоненты поля волны электрона пучка (1) и $\mathrm{HE}_{101}$-моды цилиндра (2) при резонансном взаимодействии.

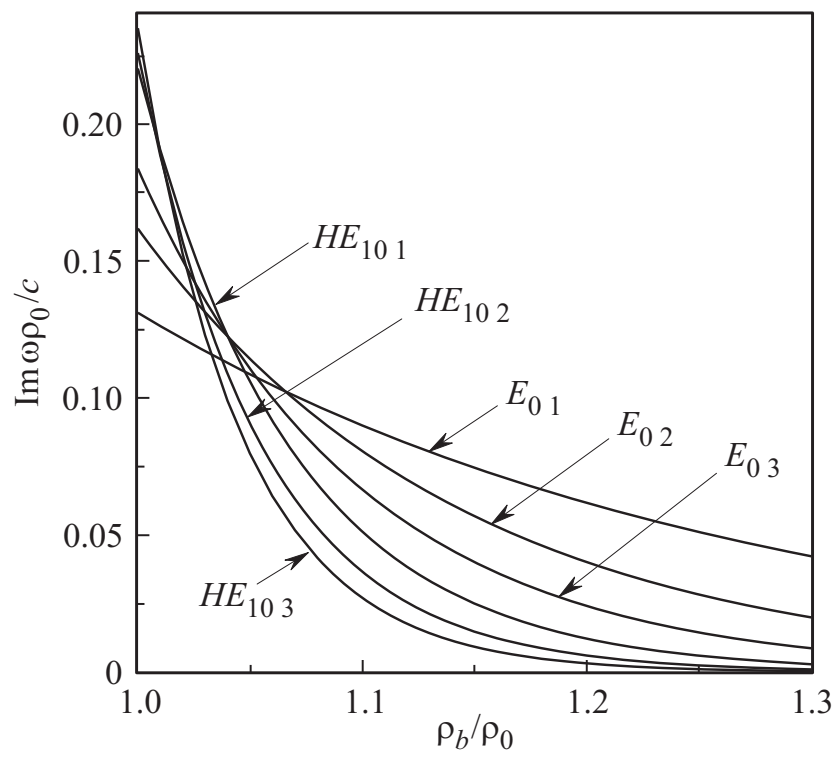

Рис. 5. Зависимости инкрементов медленных (низкочастотных) связанных волн от относительного прицельного расстояния.

$\mathrm{EH}_{10}$-мод (т. е. мод $H$-типа), а верхние $-\mathrm{HE}_{10}$-мод (E-типа). (Мы используем классификацию мод, принятую в работе [12].) Из рисунка видно, что при $q_{z}=0$

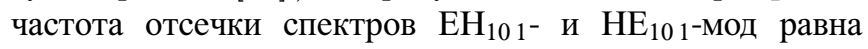
нулю.

Особый интерес представляют точки пересечения прямой $\omega \rho_{0} / c=q_{z} \beta \rho_{0}$ (т.е. $\left.\omega=q_{z} v_{0}\right)$ с ветвями собственных мод цилиндра (рис. 2). В этих точках выполняется условие фазового синхронизма (эффект Вавилова-Черенкова). Это приводит к возникновению неустойчивости в системе и нарастанию во времени амплитуд связанных волн. Резонансное взаимодействие частиц пучка с собственными модами цилиндра обоих типов приводит к „разрывам“ дисперсионных кривых взаимодействующих волн, сопровождающимся смещениями резонансных частот. При взаимодействии волн электронов пучка с симметричными модами цилиндра связанные волны возникают только при взаимодействии с собственными $E_{0 s}$-волнами (т. е. с модами $E$-типа). Поскольку при $n>0$ поля всех собственных мод цилиндра имеют отличные от нуля $E_{z n s}$-компоненты, моды как $E$-, так и $H$-типа будут взаимодействовать с волнами электронов пучка, в результате чего в системе формируются связанные волны. С одной стороны, в областях взаимодействия частиц пучка с модами цилиндра возникают устойчивые $(\operatorname{Im} \delta \omega=0)$ быстрые (высокочастотные) связанные волны. В области малых волновых чисел $\left(q_{z}<q_{z n s}\right)$ дисперсионная ветвь быстрой связанной волны соответствует собственной моде цилиндра, а в области $q_{z}>q_{z n s}$ - волне заряженной частицы пучка. С физической точки зрения данный эффект обусловлен перераспределением энергии электромагнитного поля между взаимодействующими волнами. С другой стороны, в системе в областях резонансного взаимодействия частиц пучка и собственных мод цилиндра возникают попарно медленные (низкочастотные) связанные волны, у которых $\operatorname{Im} \delta \omega \neq 0$. Как отмечено выше, одна из них является затухающей во времени, а другая - нарастающей. В области волновых чисел $q_{z}<q_{z n s}$ дисперсионная ветвь медленной связанной волны соответствует волне электрона пучка, а в области $q_{z}>q_{z n s}-$ собственной моде цилиндра. Естественно, с ростом амплитуд медленных связанных волн проявляются нелинейные эффекты, которые приводят к установлению стационарного режима. 

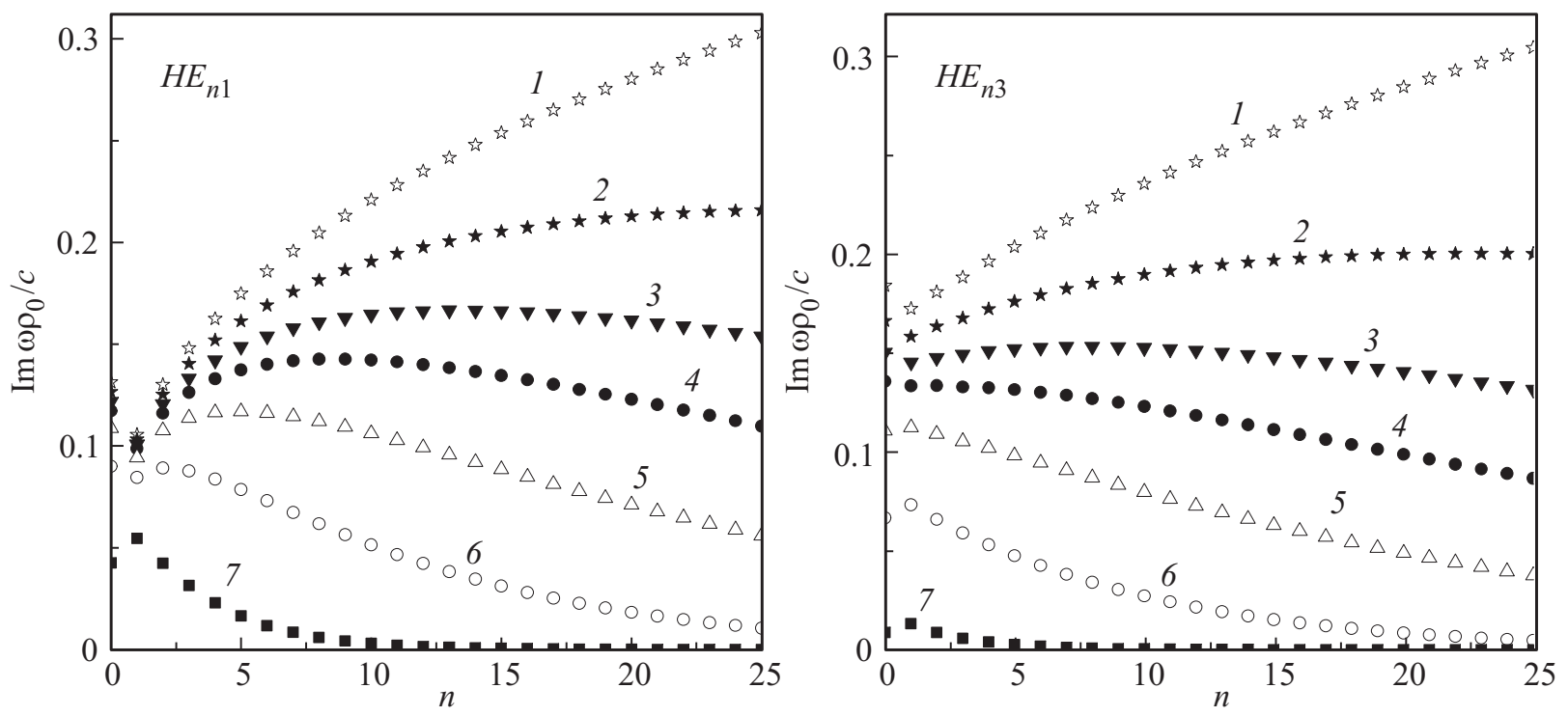

Рис. 6. Инкременты $\mathrm{HE}_{n 1}$ - и $\mathrm{HE}_{n 3}$-мод при различных относительных прицельных расстояниях между пучком и цилиндром: $\rho_{b} / \rho_{0}=1(1), 1.01(2), 1.02(3), 1.03(4), 1.05(5), 1.1(6), 1.3(7)$.

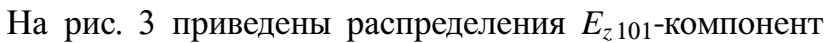
электромагнитных полей вдоль радиальной координаты в исследуемой электродинамической системе для взаимодействующих волн, которые имеют одинаковые аксиальные волновые числа, но разные частоты в соответствии с дисперсионными зависимостями. Распределения приведены вблизи области резонансного взаимодействия $\left(q_{z}<q_{z 101}\right.$ и $\left.q_{z}>q_{z 101}\right)$ и непосредственно в ней

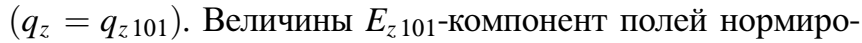
ваны на постоянную $C_{10}^{E}$, являющуюся произвольной в рамках задачи на собственные значения системы. Из рисунка видно, что вдали от резонансного взаимодействия электромагнитное поле сосредоточено внутри цилиндра вблизи его боковой поверхности и соответствует распределению поля собственной $\mathrm{HE}_{101}$-моды цилиндра. В области резонансного взаимодействия возникает связанная волна. При этом распределения полей взаимодействующих волн практически равнозначны. Электромагнитное поле сосредоточено на пучке и внутри цилиндра (рис. 4). На рис. 4 приведена топология распределений полей взаимодействующих волн в плоскости поперечного сечения рассматриваемой системы при черенковском резонансе. Значения величин $E_{z 101}$ представлены уровневой градацией белого и черного цветов. Черный и белый цвета соответствуют максимальным значениям поля, сдвинутым по фазе на $\pi$.

\section{Численный анализ инкрементов неустойчивости связанных волн}

Инкременты неустойчивости связанных волн, как было отмечено выше, оцениваются в точках пересечения дисперсионных кривых собственных мод диэлектрического цилиндра и волны электрона пучка.
На рис. 5 приведены зависимости безразмерного инкремента $\operatorname{Im} \omega \rho_{0} / c$ (т. е. $\operatorname{Im} \omega$, где $\left.\omega=\omega_{n s}+\delta \omega\right)$, взаимодействующих с электронами пучка симметричных $\mathrm{E}_{0 s}-$ и несимметричных $\mathrm{HE}_{10}$-мод, у которых радиальный индекс $s=1,2,3$, от относительного прицельного расстояния $\rho_{b} / \rho_{0}$ между пучком и цилиндром.

Отметим особенности, отображенные на рисунке. Во-первых, все зависимости являются монотонно убывающими. Во-вторых, при $\rho_{b} / \rho_{0}=1$ (пучок транспортируется вдоль боковой поверхности цилиндра) наибольшим инкрементом обладает $\mathrm{HE}_{103}$-мода, а наименьшим $E_{01}$-мода. Более того, инкремент мод с одинаковыми азимутальными индексами растет с увеличением радиального индекса. При малом прицельном расстоянии $\left(\rho_{b} / \rho_{0} \rightarrow 1\right)$ среди мод с одинаковыми радиальными индексами инкремент симметричной моды является наименьшим. В-третьих, при большом прицельном расстоянии (слабой связи) между пучком и цилиндром наибольшим значением инкремента обладает симметричная $E_{01}$-мода. Среди мод с одинаковыми азимутальными индексами наиболее неустойчивыми являются моды с радиальным индексом, равным единице.

На рис. 6 приведены инкременты $\mathrm{HE}_{n 1}$ и $\mathrm{HE}_{n 3}$-мод цилиндра в условиях резонансного взаимодействия с электронами пучка $\left(\omega_{10 s}=q_{z 10 s} v_{0}\right)$ при различных расстояниях между пучком и цилиндром. Видно, что при транспортировке пучка вдоль поверхности цилиндра $\left(\rho_{b} / \rho_{0}=1\right)$ большим инкрементом обладают высшие азимутальные моды цилиндра. С увеличением прицельного расстояния $\rho_{b} / \rho_{0}$ (уменьшением связи) между пучком и цилиндром проявляется оптимальный режим возбуждения связанной волны по максимальной неустойчивости системы. При этом азимутальный индекс собственной моды (при постоянстве радиально- 
го индекса) цилиндра с максимальным инкрементом уменьшается. В режиме максимальной неустойчивости высшие моды по радиальному индексу $s$ (но с одинаковыми индексами $n$ ) цилиндра обладают меньшим инкрементом. Следовательно, в условиях черенковского резонанса выбором прицельного расстояния $\rho_{b} / \rho_{0}$ можно осуществлять селекцию неустойчивой связанной волны системы. Кроме того, при приемлемых $\rho_{b} / \rho_{0}$ в системе осуществляется неустойчивость слабозатухающих собственных мод „шепчущей галереи“ цилиндра, взаимодействующих с резонансными частицами трубчатого пучка. Отметим, численный анализ системы показал, что при одинаковых значениях $n$ и $s$ инкременты $\mathrm{HE}_{n s}$-мод выше, чем $\mathrm{EH}_{n s}$-мод. Это свидетельствует о наиболее эффективном взаимодействии резонансных частиц пучка c собственной модой цилиндра, у которой величина компоненты $E_{z n s}$ наибольшая.

Таким образом, наиболее эффективно возбуждаются связанные волны, соответствующие резонансному взаимодействию электронов трубчатого пучка с собственными модами цилиндра $E$-типа. Выбором прицельного расстояния $\rho_{b} / \rho_{0}$ можно осуществлять селекцию возбуждаемой моды цилиндра, включая моду „шепчущей галереи“.

\section{Заключение}

В настоящей работе теоретически изучена линейная стадия неустойчивости нерелятивистского трубчатого электронного пучка, движущегося в вакууме над поверхностью диэлектрического цилиндра. Получено дисперсионное уравнение связанных волн диэлектрического цилиндра и трубчатого электронного пучка и найдены инкременты неустойчивости. Проведен численный анализ полученного дисперсионного уравнения связанных волн цилиндра и волн заряженных частиц пучка и выражения для инкрементов неустойчивости. Построены распределения продольной компоненты электрического поля в системе цилиндр-пучок, соответствующие малым окрестностям областей взаимодействия волн. Показано, что наиболее эффективно возбуждаются связанные волны, соответствующие собственным модам $E$-типа диэлектрического цилиндра с большими азимутальными индексами при малом прицельном расстоянии между электронным пучком и поверхностью цилиндра.

\section{Список литературы}

[1] Ахиезер А.И., Файнберг Я.Б. // ЖЭТФ. 1951. Т. 21. Вып. 11. C. $1262-1269$.

[2] Александров А.Ф., Богданкевич Л.С., Рухадзе А.А. Основы электродинамики плазмы. М.: Высшая школа, 1978. 407 с.

[3] Кузелев М.В., Рухадзе А.А., Стрелков П.С. Плазменная релятивистская СВЧ электроника. М.: Изд-во. МГТУ им. Н.Э. Баумана, 2002. 542 с.

[4] Энциклопедия низкотемпературной плазмы. Т. 4 / Под ред. акад. В.Е. Фортова. М.: Наука, 2000. 506 с.
[5] Дормидонтов А.В., Кириченко А.Я., Лонин Ю.Ф., Пономарев А.Г., Прокопенко Ю.В., Сотников Г.В., Уваров В.Т., Филиппов Ю.Ф. // Письма в ЖТФ. 2012. Т. 38. Вып. 2. C. $65-73$.

[6] Справочник по специальным функциям / Под. ред. М. Абрамовица, И. Стиган. М.: Наука, 1979. 832 с.

[7] Аверков Ю.О., Прокопенко Ю.В., Яковенко В.М. // Радиофизика и электроника. 2016. Т. 7 (21). Вып. 2. С. 28-35.

[8] Дормидонтов А.В., Прокопенко Ю.В., Ханкина С.И., Яковенко В.М. // Радиофизика и электроника. 2014. Т. 5 (19). Вып. 4. С. 63-72.

[9] Дормидонтов А.В., Прокопенко Ю.В., Яковенко В.М. // Радиофизика и электроника. 2015. Т. 6 (20). Вып. 4. С. 24 30.

[10] Дормидонтов А.В., Прокопенко Ю.В., Ханкина С.И., Яковенко В.М. // ЖТФ. 2015. Т. 85. Вып. 7. С. 125-132.

[11] Ведерников А.К., Говоров А.Д., Чаплик А.В. // ЖЭТФ. 2001. Т. 120. Вып. 4 (10). С. 979-985.

[12] Кириченко А.Я., Прокопенко Ю.В., Филиппов Ю.Ф., Черпак Н.T. Квазиоптические твердотельные резонаторы. Киев: Наукова думка, 2008. 286 с.

[13] Электродинамика плазмы / Под ред. А.И. Ахиезера. М.: Наука, 1974. 720 с. 